\title{
Análise de características relacionadas à variação do valor do tempo de viagem de motoristas usando técnicas de preferência declarada
}

\author{
André Nozawa Brito ${ }^{1}$; Orlando Strambi²
}

\begin{abstract}
Resumo: Através da aplicação de um procedimento empírico com técnicas de preferência declarada foi estimado o valor do tempo de viagem de motoristas de automóvel em deslocamentos rodoviários no estado de São Paulo. Foram utilizadas informações obtidas em entrevistas de preferência declarada realizadas nas principais rodovias do estado em 2005 para a estimativa de modelos de escolha discreta. Empregando-se funções de utilidade com variáveis dummy buscou-se identificar na amostra como o valor do tempo dos viajantes variava em função de características da viagem e do motorista, como faixa de renda, motivo e duração da viagem. Foi estimado o valor de $\mathrm{R} \$ 16,44 / \mathrm{h}$ para o tempo de viagem de motoristas realizando intercâmbios curtos, de até 90 minutos de duração, e R \$14,62/h para aqueles em viagens com duração entre 91 minutos e 4 horas. Dentre as diversas características analisadas, além da duração da viagem outras quatro indicaram variações significativas no valor do tempo: motorista de renda baixa, viagem com motivo lazer, motorista que informou não pagar pedágios durante a viagem realizada e sexo do motorista.
\end{abstract}

Abstract: In this paper an empirical estimation of the value of travel time of car drivers using the regional road system in the state of São Paulo is presented. Information from a roadside stated preference survey, conducted in 2005, was used for the estimation of discrete choice models. The specification of utility functions with a set of dummy variables allowed the identification of important differences in the value of travel time between segments of the sampled population characterized by a number of driver and trip attributes. Different models were estimated for different journey lengths; the value of travel time was estimated at $\mathrm{R} \$ 16.44 / \mathrm{h}$ for short trips (less than 90 minutes) and at $\mathrm{R} \$ 14.62 / \mathrm{h}$ for trips between 91 minutes and 4 hours. Other driver and trip characteristics associated with significant variation in travel time value were: low income, gender, leisure trips and trips where the driver was not paying tolls.

\section{INTRODUÇÃO}

O valor atribuído ao tempo de viagem das pessoas é um parâmetro crítico nos estudos de transportes. Seu uso encontra duas aplicações características: as avaliações econômicas de projetos e os estudos envolvendo previsões de escolha modal ou de rotas. Essas duas aplicações são respectivamente definidas por Ortúzar e Willumsen (2001) como: valor social, ou para avaliação, e valor subjetivo, ou comportamental.

Um dado que ilustra a importância do parâmetro valor do tempo nas avaliações sociais está nas estimativas de que $80 \%$ dos benefícios de um projeto de transporte sejam decorrentes de economias em tempos de viagem (Heggie, 1983; Bates e Roberts, 1986; Mackie et al., 2002). Os estudos de previsão comportamental, por sua vez, comumente envolvem modelos de simulação com redes de transporte, nos quais funções de custo generalizado medem a competitividade de uma alternativa modal ou rota. Nessas funções, o valor que se atribui ao parâmetro valor do tempo é decisivo para o resultado de uma modelagem.

\footnotetext{
${ }^{1}$ André Nozawa Brito, Desenvolvimento Rodoviário S.A. (DERSA); Escola Politécnica da Universidade de São Paulo (email: nozawabrito@gmail.com.br).

${ }^{2}$ Orlando Strambi, Escola Politécnica da Universidade de São Paulo (e-mail: ostrambi@usp.br).
}

Manuscrito recebido em 9/7/2007 e aprovado para publicação em 9/10/2007. Este artigo é parte de TRANSPORTES, volume XV, número 1, junho de 2007. ISSN: 1415-7713.

\subsection{Objetivos e estrutura do estudo}

Este estudo tem como objetivo realizar uma estimativa do valor subjetivo do tempo de viagem de motoristas em deslocamentos rodoviários e analisar como esse valor varia em função de características do motorista e da viagem. Para tanto, foi aplicado um procedimento empírico envolvendo técnicas de preferência declarada, tendo sido utilizado o conjunto de informações de uma ampla pesquisa realizada em 2005 com motoristas de automóveis nas principais rodovias do estado de São Paulo.

A estrutura deste trabalho compreende uma revisão bibliográfica sobre a teoria e procedimentos empíricos usados para a valoração do tempo de viagem e uma breve abordagem de dois temas fundamentais na metodologia adotada: i) os modelos de escolha discreta, e ii) a preferência declarada. A seguir, faz-se a descrição dos dados utilizados para as análises, passando-se à especificação dos modelos utilizados e aos resultados das estimativas. Nos itens finais são discutidos os principais resultados e as conclusões do estudo.

\section{REVISÃO TEÓRICA}

Como mencionado, o tema central deste estudo e os procedimentos utilizados compreendem três conceitos principais que serão abordados neste item: i) o valor do tempo de viagem e formas para a sua estimativa; ii) os modelos de escolha discreta, e iii) a preferência declarada. 


\subsection{Valoração do tempo}

A idéia da atribuição de um valor ao tempo gasto em qualquer atividade começou com Becker (1965), em sua teoria da alocação do tempo. Nesse estudo, o valor do tempo era descrito como o custo de oportunidade de se alocar tempo em qualquer atividade que não o trabalho, o que era justificado pelo fato de que esse tempo poderia ser convertido em dinheiro se um indivíduo passasse mais horas no trabalho. Esse princípio levava à idéia - que viria a ser superada - do valor do tempo como equivalente ao salário horário do indivíduo.

O estudo de Becker (1965) e outros que se sucederam com algumas variações, como os de Johnson (1966) e Oort (1969), tinham sua base teórica dada pelos modelos da microeconomia e no conceito da maximização da utilidade individual, segundo o qual cada indivíduo buscaria maximizar sua satisfação equilibrando o total de bens (ou dinheiro) disponíveis e o tempo destinado ao lazer, como mostrado na formulação:

$$
U=\max (G, L)
$$

em que,
$U: \quad$ utilidade ou satisfação individual;
$G$ : total de bens ou dinheiro disponí- vel;
L: $\quad$ tempo de lazer disponível.

\subsubsection{O valor subjetivo do tempo}

O chamado valor subjetivo do tempo de viagem é definido por Mackie et al. (2001) como a predisposição que um indivíduo tem de pagar pela redução de seu tempo de viagem em uma unidade. Este valor reflete a soma de pelo menos dois efeitos: i) a vontade de substituir o tempo perdido na viagem por uma atividade mais agradável ou útil, e ii) a percepção direta da duração da viagem (Jara-Díaz e Guevara, 2003). O primeiro efeito é bastante familiar: todos gostariam de usar, por exemplo, as horas perdidas no trânsito em outra atividade mais agradável ou rentável. Já a percepção da duração da viagem reflete o quanto a condição dessa é (geralmente) desagradável a um indivíduo. Pode-se ilustrar esse efeito observando-se que a predisposição de alguns indivíduos em pagar a mais para viajar na primeira classe reflete uma tentativa de minimizar o desconforto da duração da viagem. Outra evidência desse efeito é dada por inúmeros estudos que concluem que as pessoas associam diferentes níveis de desconforto para tempo de viagem em fluxo livre vis-à-vis tempo de viagem em condições de congestionamento; ou tempo da viagem dentro do ônibus vis-à-vis tempo da viagem esperando pelo ônibus no ponto.

Esses efeitos que condicionam o valor subjetivo do tempo (VST) foram matematicamente demonstrados por Jara-Díaz (2002), que partiu dos modelos de maximização da utilidade individual - mostrados no item anterior - e de premissas de um estudo clássico de Train e McFadden (1978). A equação (2) mostra a formulação deduzida por Jara-Díaz (2002) para o VST:

$$
V S T=w+\frac{\partial U / \partial W}{\partial U / \partial G}-\frac{\partial U / \partial t_{i}}{\partial U / \partial G}
$$

em que,

$$
\begin{array}{ll}
w: & \begin{array}{l}
\text { taxa de renda (salário-hora); } \\
\text { valor subjetivo do trabalho } \\
\text { puro, ou seja, os bens equiva- } \\
\text { lentes a uma unidade de tem- } \\
\text { po adicional no trabalho; }
\end{array} \\
\frac{\partial U / \partial t_{i}}{\partial U / \partial G}: \begin{array}{l}
\text { o valor subjetivo puro do } \\
\text { tempo da viagem. }
\end{array}
\end{array}
$$

Nota-se, na equação (2), que as duas primeiras parcelas do VST estão relacionadas à vontade de realocar o tempo de viagem a outras atividades. A terceira parcela refere-se à percepção da duração da viagem. Uma observação importante que se faz da formulação teórica do VST é que a única componente de mensuração direta é a taxa de renda ( $w)$, vinculada ao salário-hora das pessoas. As demais parcelas têm seu valor dado intuitivamente (ou subjetivamente) pelos indivíduos que realizam a viagem, sendo necessárias abordagens empíricas para sua estimativa (Mackie et al., 2001).

\subsubsection{A abordagem empírica}

Beesley (1965) foi pioneiro em propor um método empírico para valoração das economias de tempo de viagem. Em seu clássico estudo, apresentou o que ficou conhecido como 'Gráfico de Beesley', que consistia de um diagrama de diferenças de tempo e custo entre duas alternativas modais de transporte. Outro método usado nos anos 60 para a valoração do tempo foi denominado transfer-price, que pode ser entendido como o custo que deveria ser acrescido a uma alternativa para tornar sua atratividade igual a uma outra (Ortúzar e Willumsen, 2001). Envolvia, por exemplo, perguntar ao entrevistado quanto a tarifa de seu modo preferido de transporte deveria aumentar para fazê-lo mudar para uma outra alternativa. Esse método, a exemplo do proposto por Beesley, baseava suas estimativas em análises de regressão linear.

A evolução nos estudos de valoração do tempo foi trazida pela aplicação dos modelos de escolha discreta, a partir dos anos 70. Essa abordagem, ao contrário das anteriores, contempla o fato de que o processo de medição das escolhas é sujeito a erros aleatórios, como será descrito no item 2.2 a seguir. Inicialmente, os modelos eram estimados com informações de prefe- 
rência revelada, geralmente observações das escolhas feitas (Hensher e Truong, 1985). O problema da limitação que esse tipo de informação oferecia - tema discutido a seguir - foi em grande parte solucionado na década de 80 com a incorporação de técnicas de preferência declarada.

\subsection{Modelos de escolha discreta}

Modelos de escolha discreta são usados para a estimativa de funções de utilidade condicionais, que descrevem a preferência do consumidor face ao conjunto de atributos observados. A utilidade pode ser definida como uma medida de atratividade de uma alternativa em relação às demais pertencentes a um mesmo conjunto de escolha. Dentro do conceito da utilidade aleatória, a utilidade de uma alternativa $j$ para um indivíduo $n\left(U_{n j}\right)$ é formada por uma parte observável, ou mensurável, e uma parte não observável, como descrito a seguir:

$$
U_{n j}=V_{n j}+\varepsilon_{n j}
$$

A parte observável $V_{n j}$ envolve usualmente atributos como tempos e custos de viagem de uma alternativa $j$. A parcela $\varepsilon_{n j}$, também denominada termo de erro aleatório, reflete as idiossincrasias e gostos particulares de cada indivíduo, juntamente com os erros de observações e de medidas incorridos durante a modelagem (Ortúzar e Willumsen, 2001). A distribuição que se admite para esse termo de erro leva a diferentes tipos de modelo de escolha, como logit, probit ou mixed logit, sendo o primeiro o mais amplamente usado. Em um modelo logit a probabilidade de escolha de uma alternativa $i$ (dentre um conjunto de $j$ possíveis) para um indivíduo $n$ é dada por:

$$
P_{n i}=\frac{e^{V_{n i}}}{\sum_{j} e^{V_{n j}}}
$$

\subsection{Preferência declarada}

Para o campo das aplicações em transporte, Kroes e Sheldon (1988) definem que o termo preferência declarada (PD) refere-se a uma família de técnicas que usam declarações de indivíduos sobre suas preferências em um conjunto de opções de transporte para a estimativa de funções de utilidade.

Na estimativa de modelos de escolha, o uso de informações obtidas por PD apresenta uma série de vantagens em relação à forma tradicional de obtenção de dados através da observação ou do questionamento sobre as escolhas feitas pelas pessoas, denominada preferência revelada (PR). Entre os benefícios do uso da PD pode-se citar (Morikawa, 1989): i) o conjunto de escolha pode ser pré-especificado, descrevendo situações existentes ou cenários hipotéticos construídos pelo pesquisador; ii) o intervalo de variação dos atributos pode ser estendido; iii) há a possibilidade de controle da correlação entre os atributos; iv) atributos de difícil observação, como confiabilidade e segurança, podem ser incorporados à pesquisa, e v) possibilidade de obtenção de múltiplas observações para um único entrevistado. As informações obtidas por PD apresentam, no entanto, menor confiabilidade e validação do que aquelas obtidas por PR. Entende-se por confiabilidade a idéia de que repetidas medições de uma escolha levarão a resultados similares. A validação diz respeito à relação entre o que se observou ser escolhido e o que foi realmente escolhido, ou seja, nem sempre o que as pessoas declaram que escolheriam em uma situação hipotética será a sua escolha quando aquela situação for real.

Na concepção de um experimento de PD o aspecto central é o desenho do experimento, que envolve a observação do efeito sobre uma variável, chamada variável resposta, em função da manipulação dos níveis de outras variáveis. Esse aspecto de relativa complexidade é abordado de forma didática por Hensher et al. (2005).

\section{DADOS UTILIZADOS}

A pesquisa cujo conjunto de informações foi utilizado para as análises foi realizada em 2005 pela Secretaria dos Transportes do Estado de São Paulo. O levantamento compreendeu 28 pontos de pesquisa distribuídos na malha rodoviária principal - pedagiada e não pedagiada - do estado, tendo sido geradas 8.256 entrevistas com motoristas de automóveis.

As entrevistas, feitas com computadores portáteis do tipo "palm top", consistiram de uma parte de PR e outra de PD. Na primeira, foram levantadas informações gerais sobre o motorista e a viagem realizada. A parte da PD consistiu da aplicação de questões nas quais o entrevistado deveria escolher entre duas alternativas rotuladas para um trecho de sua viagem: uma rota pedagiada e uma rota não pedagiada. As alternativas eram caracterizadas por seus tempos e custos tarifários, sendo pedido ao entrevistado que considerasse ambas as rotas em iguais condições de pavimento.

O experimento considerou 3 atributos: i) o tempo de viagem pela rota pedagiada, ii) o custo tarifário da rota pedagiada, e iii) o tempo de viagem pela rota não pedagiada. Adotaram-se 3 níveis para cada atributo, de acordo com a Tabela 1 a seguir:

Na Tabela 1, $T$ representa o tempo de viagem informado pelo entrevistado e $P$ o valor da tarifa de pedágio, definido em $\mathrm{R} \$ 5,00$ quando o posto de pesquisa se situava em rodovia de pista simples e $\mathrm{R} \$ 7,50$ quando em pista dupla. $\mathrm{O}$ valor $P$ da tarifa podia ser alterado pelo analista durante a execução da pesquisa 
Tabela 1. Valores associados aos níveis dos atributos

\begin{tabular}{cccc}
\hline & \multicolumn{3}{c}{ Atributo } \\
\cline { 2 - 4 } Nível & $\begin{array}{c}\text { Tempo de viagem } \\
\text { pela rota } \\
\text { pedagiada (min) }\end{array}$ & $\begin{array}{c}\text { Custo do } \\
\text { pedágio } \\
(\mathbf{R} \$)\end{array}$ & $\begin{array}{c}\text { Tempo de viagem } \\
\text { com atraso pela rota } \\
\text { não pedagiada (min) }\end{array}$ \\
\hline-1 & $T-15$ & $P-30 \%$ & $T+28$ \\
0 & $T$ & $P$ & $T+40$ \\
+1 & $T+15$ & $P+30 \%$ & $T+52$ \\
\hline
\end{tabular}

de modo a ajustar a sensibilidade do experimento. Foi utilizado um desenho fatorial fracional gerando nove conjuntos de escolha binária que eram apresentados a cada entrevistado (Brito, 2007).

\section{ESTIMATIVAS DOS MODELOS DE ESCOLHA}

As análises desenvolvidas utilizaram modelos do tipo logit multinomial. A especificação das funções de utilidade indireta seguiu a forma aditiva e linear nos parâmetros que, de acordo com Gunn (2000), é a mais comumente utilizada para caracterizar a atratividade de uma alternativa em estudos visando estimativas do valor do tempo.

Para a identificação da influência de algumas características dos motoristas e da viagem na variação do valor do tempo foram empregadas funções de utilidade com variáveis dummies representando as características a serem analisadas.

A forma adotada para as funções, mostrada nas equações 5 e 6 a seguir, representa uma generalização daquela proposta por Bradley e Gunn (1990):

$$
\begin{gathered}
U_{\text {pedi }}=\alpha_{0}+\left(\beta_{0}+\sum_{k} \beta_{k} \cdot \delta_{i k}\right) \cdot c_{\text {pedi }}+\left(\gamma_{0}+\sum_{k} \gamma_{k} \cdot \delta_{i k}\right) \cdot t_{\text {pedi }} \\
U_{\text {npedi }}=\left(\theta_{0}+\sum_{k} \theta_{k} \cdot \delta_{i k}\right) \cdot t_{\text {npedi }}
\end{gathered}
$$

em que,

$U_{\text {pedi }}$ : utilidade da alternativa pedagiada para um indivíduo $i$;

$U_{\text {npedi }}$ : utilidade da alternativa não pedagiada para um indivíduo $i$;

$C_{\text {pedi }}$ : custo tarifário pela alternativa pedagiada para um indivíduo $i$ (centavos);

$t_{\text {pedi }}$ : tempo de viagem pela alternativa pedagiada para um indivíduo $i$ (minutos);

$t_{\text {npedi }}$ : tempo de viagem pela alternativa não pedagiada para um indivíduo $i$ (minutos);

$\alpha_{0}$ : termo principal da constante específica da alternativa pedagiada;

$\beta_{0}, \gamma_{0}$ : coeficientes principais de custo e tempo pela alternativa pedagiada;

$\theta_{0}$ : coeficiente principal do tempo pela alternativa não pedagiada; $\beta_{k}, \gamma_{k}: \quad$ coeficientes diferenciais de custo e tempo pela alternativa pedagiada para a categoria $k$ representada por variáveis dummies;

$\theta_{k}$ : coeficiente diferencial de tempo pela alternativa não pedagiada para a categoria $k$ representada por variáveis dummies;

$\delta_{i k}$ : variáveis dummies (0/1) indicando pertinência ou não do indivíduo $i$ à categoria $k$ considerada.

Nas funções de utilidade indireta, o valor do tempo $(V T)$ corresponde à taxa entre a utilidade marginal do tempo de viagem $\frac{\partial U}{\partial t}$ e a utilidade marginal do custo de viagem $\frac{\partial U}{\partial c}$ (Jara-Díaz, 2000). De forma análoga à equação (5), sendo $\beta$ o coeficiente do custo de viagem e $\gamma$ o do tempo, tem-se:

$$
V T=\frac{\frac{\partial U}{\partial t}}{\frac{\partial U}{\partial c}}=\frac{\gamma}{\beta}
$$

Na forma com dummies aqui adotada para as funções de utilidade, o valor do tempo de viagem para um indivíduo $i$ que pertença a um segmento $k$ será dado por:

$$
V T_{i}=\frac{\gamma_{0}+\sum_{k} \gamma_{k} \cdot \delta_{i k}}{\beta_{0}+\sum_{k} \beta_{k} \cdot \delta_{i k}}
$$

Cabe observar que a equação (8) expressaria o valor do tempo para indivíduos que usam a rota pedagiada. No entanto, os testes estatísticos realizados após a estimativa dos modelos indicaram não haver diferenças significativas entre os coeficientes do tempo pelas alternativas pedagiada e não pedagiada, de forma que o VT para um indivíduo $i$ pode ser interpretado independentemente da alternativa escolhida por esse.

\subsection{Variáveis analisadas}

Os modelos estimados para análise da variação do valor do tempo consideraram as seguintes variáveis: i) posição de chefe da família; ii) sexo do motorista; iii) faixa de renda familiar; iv) viagem realizada durante o período de pico da manhã (entre 06h00min e 08h30min); v) pagamento de pedágios durante a viagem; vi) motivo da viagem (trabalho diário, negócios ou outros), e vii) viagem com motivo lazer.

A característica descrevendo a faixa de renda familiar mensal considerou quatro categorias: i) baixa (até R\$ 900); ii) média (de R \$ 901 a R\$ 3000); iii) médiaalta (de R\$ 3001 a R\$ 6000), e iv) alta (acima de R\$ 6000).

A variável motivo da viagem foi analisada em sua segmentação usualmente adotada na literatura, sendo 
o ‘trabalho diário' o motivo que mais de aproxima do definido em inglês como 'commuter'. Adicionalmente, foi inserida uma variável para a verificação do motivo lazer, muitas vezes não contemplado nos estudos. Com relação ao pagamento ou não de pedágios, foi usada uma informação dada pelo próprio motorista.

Nas estimativas, a amostra foi separada em dois grupos: i) viagens curtas, com duração de até 1 h e 30 minutos, e ii) viagens longas, com duração entre 1h 31minutos e 4 horas. Tal segmentação foi adotada após análises preliminares que indicaram haver diferenças importantes no valor do tempo segundo essa variável.

\subsection{Resultados dos modelos}

Como é comum em levantamentos de PD, mais de um terço das entrevistas não foram utilizadas nas estimativas dos modelos por conter respostas onde o motorista não ponderava os atributos apresentados de forma a maximizar sua utilidade. Cita-se como exemplo as entrevistas onde o respondente concentrava todas as suas escolhas um uma mesma alternativa - seja pedagiada ou não pedagiada.

As estimativas dos modelos de escolha foram realizadas com o software de licença livre Biogeme (Bierlaire, 2005), que utiliza o método da máxima verossimilhança para a estimativa dos parâmetros.

Inicialmente, foram estimados os modelos com dummies testando individualmente cada uma das 7 características analisadas, citadas no item 4.1. Para aquelas indicando posição de chefe de família, faixa de renda familiar diferente de baixa, realização de viagem no pico da manhã e motivo da viagem, classificado entre trabalho diário, negócios e outros, os valores dos coeficientes adicionais de tempo e custo não foram estatisticamente significativos. Ou seja, tais características não indicaram em variações comportamentais significativas nos modelos estimados.

A Tabela 2, a seguir, mostra o resultado dos modelos estimados apenas com as características cujos coeficientes adicionais de tempo e custo foram estatisticamente significativos. Essas características foram: i) motorista de renda familiar baixa; ii) não pagamento de pedágios na viagem; iii) viagem com motivo lazer, e iv) motorista do sexo feminino.

Observa-se nos modelos que todos os coeficientes principais estimados, bem como as constantes específicas, foram significativos no nível de significância de 0,05 ( $\mathrm{t}=1,96)$. Os modelos estimados apresentaram valores do rho-quadrado em torno de 0,43 , indicando boa aderência aos dados e os valores da estatística da taxa de verossimilhança foram bastante superiores ao valor do qui-quadrado crítico. Nota-se também que os coeficientes adicionais do tempo de viagem não pedagiado não estão apresentados na Tabela 2, por serem
Tabela 2. Resultados dos modelos estimados

\begin{tabular}{|c|c|c|}
\hline Variáveis & $\begin{array}{c}\text { Viagens com } \\
\text { duração até } 90 \\
\text { min }\end{array}$ & $\begin{array}{c}\text { Viagens com } \\
\text { duração entre } \\
91 \text { min e } 4 h \\
\end{array}$ \\
\hline \multicolumn{3}{|l|}{ Coeficientes principais: } \\
\hline Cte. Específica $\left(\alpha_{0}\right)$ & $\begin{array}{l}-0,8116 \\
(-7,3176)\end{array}$ & $\begin{array}{l}-0,5161 \\
(3,0051)\end{array}$ \\
\hline Custo tarifário $\left(\beta_{0}\right)$ (cent) & $\begin{array}{c}-0,0059 \\
(-33,5860)\end{array}$ & $\begin{array}{c}-0,0065 \\
(-25,2579)\end{array}$ \\
\hline $\begin{array}{l}\text { Tempo de viagem } \\
\text { pedagiado }\left(\gamma_{0}\right)(\mathrm{min})\end{array}$ & $\begin{array}{c}-0,1588 \\
(-72,8549)\end{array}$ & $\begin{array}{c}-0,1585 \\
(-50,9747)\end{array}$ \\
\hline $\begin{array}{l}\text { Tempo de viagem } \\
\quad \text { não pedagiado }\left(\theta_{0}\right)(\mathrm{min})\end{array}$ & $\begin{array}{c}-0,1594 \\
(-64,1390)\end{array}$ & $\begin{array}{c}-0,1583 \\
(-49,7131)\end{array}$ \\
\hline \multicolumn{3}{|c|}{ Coeficientes adicionaisdas dummies $\left(\beta_{k}\right.$ e $\left.\gamma_{k}\right)$ : } \\
\hline $\begin{array}{l}\text { Custo tarifário } \\
\text { se renda baixa }\end{array}$ & $\begin{array}{l}-0,0015 \\
(-3,8285)\end{array}$ & $\begin{array}{c}-0,0006 \\
(-0,9990)\end{array}$ \\
\hline $\begin{array}{l}\text { Tempo de viagem pedagiado } \\
\text { se renda baixa }\end{array}$ & $\begin{array}{c}0,0038 \\
(0,7797)\end{array}$ & $\begin{array}{c}0,0063 \\
(0,82740)\end{array}$ \\
\hline $\begin{array}{l}\text { Custo tarifário se não } \\
\text { pagante de pedágio }\end{array}$ & $\begin{array}{l}-0,0006 \\
(-2,0035)\end{array}$ & $\begin{array}{c}-0,0016 \\
(-2,52830)\end{array}$ \\
\hline $\begin{array}{l}\text { Tempo de viagem pedagiado } \\
\text { se não pagante de pedágio }\end{array}$ & $\begin{array}{l}-0,0073 \\
(-1,9796)\end{array}$ & $\begin{array}{c}0,0057 \\
(0,85246)\end{array}$ \\
\hline Custo tarifário se viagem a lazer & $\begin{array}{c}0,0013 \\
(2,2846)\end{array}$ & $\begin{array}{c}0,0007 \\
(1,31058)\end{array}$ \\
\hline $\begin{array}{l}\text { Tempo de viagem pedagiado } \\
\text { se viagem a lazer }\end{array}$ & $\begin{array}{l}-0,0206 \\
(-3,1176)\end{array}$ & $\begin{array}{c}-0,0155 \\
(-2,16311)\end{array}$ \\
\hline Custo tarifário se sexo feminino & $\begin{array}{c}0,0003 \\
(0,7136)\end{array}$ & $\begin{array}{c}-0,0007 \\
(-1,02415)\end{array}$ \\
\hline $\begin{array}{l}\text { Tempo de viagem pedagiado } \\
\text { se sexo feminino }\end{array}$ & $\begin{array}{c}0,0007 \\
(1,4535)\end{array}$ & $\begin{array}{c}-0,0414 \\
(-4,06677)\end{array}$ \\
\hline \multicolumn{3}{|l|}{ Estatísticas } \\
\hline Observações & 29952 & 14247 \\
\hline$l^{*}(0)$ & -20761 & -9875 \\
\hline$l^{*}(\theta)$ & -11804 & -5562 \\
\hline$-2\left(l^{*}(0)-l^{*}(\theta)\right)$ & 17913 & 8625 \\
\hline$\rho^{2}$ & 0,4314 & 0,4367 \\
\hline$\rho_{\text {ajust }}^{2}$ & 0,4304 & 0,4347 \\
\hline
\end{tabular}

estatisticamente iguais aos do tempo pedagiado.

Com relação aos coeficientes adicionais das dummies estimados, observa-se que aqueles relativos ao custo para motoristas de renda familiar baixa tiveram valor negativo, indicando que motoristas pertencentes a esse segmento percebem maior desutilidade no pagamento de tarifas, como esperado. $\mathrm{O}$ mesmo se verifica para as pessoas que informaram não pagar nenhum pedágio em sua viagem. Para os viajantes a lazer foram estimados termos adicionais de custo positivos, indicando que esse grupo associa menor desutilidade ao pagamento do pedágio. Além disso, os coeficientes adicionais relativos ao tempo de viagem são negativos, ou seja, esses motoristas percebem maior desconforto na duração da viagem. Esses dois aspectos contribuem para que esses motoristas tenham valores de tempo bastante superiores aos demais. Em motoristas do sexo feminino, os coeficientes adicionais do tempo de viagem foram consideravelmente altos e negativos 
no modelo das viagens com duração superior a $1 \mathrm{~h}$ e 30min, indicando que em deslocamentos mais longos as mulheres motoristas percebem maior desconforto do que os homens na duração da viagem.

A Tabela 3 mostra os valores do tempo calculados para cada um dos grupos contidos nos modelos estimados.

A categoria base indicada na Tabela 3 é representada por um motorista homem de renda não baixa, realizando um deslocamento não a lazer e que paga ao menos um pedágio em sua viagem.

\section{OBSERVAÇÕES SOBRE OS RESULTADOS}

Neste item são feitas observações sobre as principais características que implicaram em variações no valor do tempo de viagem nos modelos estimados.

\subsection{Renda do motorista}

Com relação aos resultados obtidos, descritos na Tabela 3 , verifica-se que aos motoristas de renda baixa

Tabela 3. Valores do tempo de viagem estimados

\begin{tabular}{|c|c|c|}
\hline Categoria & $\begin{array}{l}\text { Viagens com } \\
\text { duração de } \\
\text { até } 90 \mathrm{~min} \\
(\mathrm{R} \$ / \mathrm{h}) \\
\end{array}$ & $\begin{array}{c}\text { Viagens com } \\
\text { duração entre } \\
91 \text { min e } 4 h \\
(\mathrm{R} \$ / h)\end{array}$ \\
\hline Base & 16,44 & 14,62 \\
\hline $\begin{array}{l}\text { Renda baixa } \\
\text { (diferença p/ base) }\end{array}$ & $\begin{array}{c}12,75 \\
(-22,5 \%)\end{array}$ & $\begin{array}{c}12,85 \\
(-12,1 \%)\end{array}$ \\
\hline $\begin{array}{l}\text { Não pagante de pedágios } \\
\text { (diferença p/ base) }\end{array}$ & $\begin{array}{c}15,58 \\
(-5,2 \%)\end{array}$ & $\begin{array}{c}11,31 \\
(-22,6 \%)\end{array}$ \\
\hline $\begin{array}{l}\text { Viagem a lazer } \\
\text { (diferença p/ base) }\end{array}$ & $\begin{array}{c}23,93 \\
(+45,6 \%)\end{array}$ & $\begin{array}{c}17,99 \\
(+23,0 \%)\end{array}$ \\
\hline $\begin{array}{l}\text { Sexo feminino } \\
\text { (diferença p/ base) }\end{array}$ & $\begin{array}{c}17,26 \\
(+5,0 \%)\end{array}$ & $\begin{array}{c}16,65 \\
(+13,9 \%)\end{array}$ \\
\hline
\end{tabular}

foram estimados valores de tempo 22,5\% inferiores aos da categoria base, no grupo das viagens curtas. Nota-se, pelos coeficientes apresentados na Tabela 2, que para as viagens de longa duração essa diferença não foi significativa. Como mencionado, dentre os motoristas pertencentes às demais faixas definidas como 'média', 'média-alta' e 'alta', não se verificaram diferenças comportamentais. A baixa eficiência da informação sobre a faixa de renda do motorista em indicar variações no valor do tempo, o que não raro é verificada em outros estudos, como os citados por Van Zyl e Raza (2006). Nos casos como os deste estudo, onde a informação sobre renda salarial é fornecida pelo próprio motorista, paira a suspeita de que as pessoas com renda mais alta tenham tendência a informar salários abaixo dos que realmente ganham e, por outro lado, aquelas com salários muito baixos, por constrangimento, venham a informar valores superiores. Neste estudo uma análise exploratória buscando pro- xies para a renda indicou que entrevistados com posse de 3 ou mais veículos teriam valores de tempo cerca de $60 \%$ superiores. Tal resultado foi significativo apenas no segmento das viagens com duração superior a 1h e 30 min.

\subsection{Pagamento de pedágios na viagem}

Os motoristas que declararam não pagar nenhum pedágio na realização de sua viagem tiveram valores de tempo 22,6\% inferiores, quando a viagem tem longa duração (superior a $1 \mathrm{~h}$ e $30 \mathrm{~min}$ ), e apenas 5,2\% inferiores, nas viagens curtas. A explicação para essa diferença pode estar no fato de que, para as viagens mais curtas, uma parcela dos motoristas que afirmou não pagar nenhum pedágio não estaria, na verdade, fazendo algum esforço para evitar esse pagamento: simplesmente sua rota natural não cruzaria nenhuma praça de pedágio. Já, por outro lado, em uma viagem com duração entre 1 hora e meia a 4 horas seria muito mais provável que o motorista cruzasse alguma praça de pedágio. E, nesse segmento, dentre os motoristas que declararam realizar a viagem sem pagar nenhum pedágio, provavelmente uma grande parcela está fazendo um esforço para evitar as cobranças. Assim, a informação que permite identificar a diferença de comportamento dos não pagantes de pedágio é provavelmente mais consistente no segmento das viagens com duração entre 91 e 240 minutos.

\subsection{Motivo de viagem trabalho diário e negócios}

Uma conclusão deste estudo que poderia ser considerada inesperada está na variação do valor do tempo em função do motivo da viagem. Observou-se que a segmentação considerando os motivos 'trabalho diário', 'negócios' e 'outros' - comumente adotada na bibliografia, e onde tradicionalmente o grupo de viajantes a negócios tem os maiores valores estimados - não revelou diferenças importantes nas estimativas aqui realizadas. Uma provável explicação para a diferença não significativa entre o comportamento dos viajantes ao trabalho diário e a negócios na amostra utilizada é dada por Bradley e Gunn (1990), que reconhecem haver no motivo negócios a dificuldade de distinção entre dois subgrupos que supostamente teriam valores de tempo bastante distintos: i) a figura do tradicional executivo de negócios, e ii) o trabalhador que está meramente viajando a um local não fixo de trabalho, como um representante de vendas, por exemplo. No caso aqui estudado, os pontos de pesquisa inseriam-se predominantemente em regiões não urbanas e em rodovias de característica rural, onde as viagens amostradas provavelmente reuniam um conjunto bastante heterogêneo de viajantes a negócios, onde muitos se enquadrariam no segundo grupo citado por Bradley e Gunn (1990). 


\subsection{Viagens com motivo lazer}

Com relação às viagens a lazer observaram-se valores de tempo $45,6 \%$ superiores no caso de viagens curtas e $23 \%$ superiores para viagens longas. Sobre esse resultado, Schmitz (2001), em estudo semelhante em Florianópolis, identificou também para motoristas turistas valores de tempo bastante superiores aos dos moradores locais. Um aspecto a ser observado nas viagens a lazer é que essas são, caracteristicamente, de menor freqüência do que as por outros motivos. Assim, esses viajantes teriam menor conhecimento da região e de alternativas de rotas existentes preferindo manter-se em uma via pedagiada, que proporcionaria maior sensação de segurança e definição mais clara de para onde está levando o motorista (Hensher e Goodwin, 2004).

\subsection{Sexo do motorista}

Motoristas do sexo feminino, especialmente aquelas realizando deslocamentos com duração entre 91 minutos e 4 horas, tiveram maiores valores de tempo por perceberem maior desutilidade na duração da viagem do que os homens, como se observa na Tabela 2. Nas viagens curtas, de até 90 minutos, essa maior desutilidade para o tempo de viagem, em relação à categoria base dada pelos homens, não foi significativa. Uma possível interpretação do resultado é que as mulheres motoristas percebam nas viagens longas algum tipo de desconforto que não associam às mais curtas, talvez por motivos relativos à segurança.

\subsection{Duração da viagem}

Por fim, a duração da viagem foi identificada como uma importante variável associada a variações no valor do tempo dos viajantes. A questão da variação do valor do tempo dos motoristas em função da duração da viagem em escolhas entre rotas pedagiadas e não pedagiadas é amplamente analisada na literatura. Os resultados e conclusões encontrados, no entanto, não são todos convergentes. Enquanto Steimetz e Brownstone (2005), Frejinger et al. (2006) e Jiang e Morikawa (2004) descreveram que os valores do tempo decrescem na medida em que a duração da viagem aumenta - resultado verificado no presente estudo - outros como Van Zyl e Raza (2006) e Mackie et al. (2003) concluíram que motoristas em viagens longas associariam maiores valores a seu tempo. Mackie et al. (2003), no entanto, reconhecem haver algumas reservas com relação à consistência de seus resultados face à teoria microeconômica e defendem que há necessidade de maiores investigações sobre os efeitos da duração no valor do tempo de viagem.

\section{CONCLUSÕES}

Os resultados deste trabalho, em que foram apresenta- dos valores do tempo variando de $\mathrm{R} \$ 11,31 / \mathrm{h}$ a $\mathrm{R} \$ 23,93 / \mathrm{h}$, indicam que a diversidade nos valores de tempo de viagem é um importante aspecto a ser considerado nos estudos que envolvem previsão do comportamento da demanda. Por exemplo, o pedagiamento de uma rota provocará diferentes reações nos diversos tipos de viajantes e de viagens que a utilizam. E essas diferenças serão prioritariamente condicionadas por diferentes predisposições em pagar por reduções no tempo de viagem. Assim, existindo informação em nível confiável, estudos que envolvam previsões comportamentais terão importantes ganhos de precisão de resultados se considerarem a segmentação da demanda segundo variáveis de maior relevância na variação do valor do tempo.

Com relação a características que influem na variação do valor do tempo, deve-se sempre lembrar que o problema da estimativa do valor do tempo é bastante específico ao contexto analisado (Gunn, 2000). Observou-se aqui que a tradicional segmentação por motivo da viagem entre trabalho diário e negócios, muito utilizada em estudos de característica urbana, não indicou variações significativas neste estudo, que envolvia predominantemente deslocamentos rodoviários regionais.

\section{REFERÊNCIAS BIBLIOGRÁFICAS}

Bates, J. e Roberts, M. (1986) Value of Time Research: Summary of Methodology and Findings. Anais do 14th PTRC Summer Annual Meeting, University of Sussex, Inglaterra.

Becker, G. (1965) A Theory of the Allocation of Time. Economic Journal, 75, pp 493-517.

Beesley, M.E. (1965) The Value of Time Spent in Travelling: Some New Evidence. Economica, vol 32, n. 126, pp. 174-185.

Ben-Akiva, M. e Lerman, S. R. (1985) Discrete Choice Analysis - Theory and Application to Travel Demand. MIT Press, Cambridge.

Bierlaire, M. (2005) An Introduction to Biogeme (Version 1.4). Disponível em: <http://roso.epfl.ch/mbi/biogeme/doc/ tutorial.pdf>. Acessado em fevereiro de 2006.

Bradley, M.A. e Gunn, H.F. (1990) Stated Preference Analysis of Values of Travel Time in the Netherlands. Transportation Research Record, 1285, pp 78-88.

Brito, A.N. (2007) Aplicação de um Procedimento usando Preferência Declarada para a Estimativa do Valor do Tempo de Viagem de Motoristas em uma Escolha entre Rotas Rodoviárias Pedagiadas e não Pedagiadas. Dissertação de Mestrado, Escola Politécnica da Universidade de São Paulo

Frejinger, E., Bierlaire, M., Stojanovic, J., Vrtic, M., Schuessler, N. e Axhausen, K.W. (2006) A Route Choice Model in Switzerland Based on RP and SP Data. Paper. Transport and Mobility Laboratory, Ecole Polytechnique Fédérale de Lausanne. Switzerland, Lausanne. Disponível em: <http://www.e-collection.ethbib.ethz. ch/show?type=incoll\&nr=1098\&part=text $>$. Acessado em novembro de 2006.

Gunn, H.F. (2000) An Introduction to the Valuation of Travel-Time Savings and Losses. In Hensher, D.A. e Button, K.J. (eds) Handbook of Transport Modelling, Elsevier, Oxford.

Hensher, D.A. (2001) Measurement of the Valuation of Travel Time Savings. Journal of Transport Economics and Policy, vol 35, part I, pp 71-98.

Hensher, D.A. e Goodwin, P. (2004) Using Values of Time Savings for Toll Roads: Avoiding Some Common Errors. Transport Policy, 11, pp 171-181.

Hensher, D.A.; Rose, J.M. e Greene, W.H. (2005) Applied Choice Analysis - A Primer. Cambridge University Press, New York. 
Hensher, D.A. e Truong, P.T. (1985) Valuation of Travel Time Savings A Direct Experimental Approach. Journal of Transport Economics and Policy, pp 237-261.

Heggie, I.G. (1983) Valuing Savings In Non Working Travel Time: The Empirical Dilema. Transportation Research A, 17, pp 13-23.

Jara-Díaz, S.R. (2000) Allocation and Valuation of Travel-Time Savings. In Hensher, D.A.; Button, K.J. (eds) Handbook of Transport Modelling, Elsevier, Oxford.

Jara-Díaz, S.R. (2002) The Goods / Activities Framework For Discrete Travel Choices: Indirect Utility And Value Of Time. In Mahmassani, H.S. (ed) In Perpetual Motion - Travel Behaviour Research Opportunities And Application Challenges. Elsevier, Netherlands.

Jara-Díaz, S.R. e Guevara, C.A. (2003) Behind the Subjetive Value of Travel Time Savings - The Perception of Work, Leisure, and Travel from a Joint Mode Choice Activity Model. Journal of Transport Economics And Policy, vol 37, part 1, pp 29-46.

Jiang, M. e Morikawa, T. (2004) Theoretical Analysis on the Variation of Value of Travel Time Savings. Transportation Research A, 38, pp 551-571.

Johnson, M. (1966) Travel Time and the Price of Leisure. Western Economics Journal $\mathrm{n}^{\circ} 4$.

Kroes, E.P. e Sheldon, R.J. (1988) Stated Preference Methods - An Introduction. Journal of Transport Economics and Policy, vol 22, pp 11-25.

Mackie, P.J.; Jara-Díaz, S.R. e Fowkes, A.S. (2001) The Value of Travel Savings in Evaluation. Transportation Research E, 37, Elsevier, UK.

Mackie, P.J.; Grant-Muller, S.; Nellthorp, J. e Pearman, A. (2002) SocioEconomic Cost Benefit Analysis For Transport Infrastructure Project Appraisal. United Nations - Economic Commission For Europe Inland Transport Committee, Geneva. Disponível em:<http://www.unece.org/trans/doc/2002/wp5/TRANS-WP52002-07e.pdf>, acessado em: 03/2006.

Mackie P.J., Fowkes A.S., Wardman M., Whelan G., Nellthorp J. e Bates J. (2003) Valuation of Travel Time Savings in the UK - Summary Report to the Department for Transport. Institute for Transport Studies Working Paper, University of Leeds.

Morikawa, T. (1989) Incorporating Stated Preference Data in Trave Demand Analysis. Doctoral Dissertation - Massachusetts Institute of Technology, Cambridge.

Oort, C.J. (1969) The Evaluation of Travelling Time. Journal of Transport Economics and Policy, 3, 279-286.

Ortúzar, J.D. e Willumsen L.G. (2001) Modelling Transport. John Wiley \& Sons, $3^{\mathrm{a}}$ ed, New York

Schmitz, R. (2001) Uma Contribuição Metodológica para Avaliação da Tarifa de Pedágio em Rodovias. Tese de Doutorado, Engenharia de Produção UFSC, Florianópolis.

Steimetz S.S.C e Brownstone D. (2005) Estimating Commuters' "Value of Time” with Noisy Data: a Multiple Imputation Approach. Transportation Research B, 39, pp 865-889.

Train, K. e McFadden, D. (1978) The Goods/Leisure Tradeoff and Disaggregate Work Trip Mode Choice Models. Transportation Research, vol 12, pp 349-353.

Van Zyl, N. J. W. e Raza, M. (2006) In Search of the Value of Time: From South Africa to India. In Stopher, P., Stecher, C. (eds) Travel Survey Methods - Quality and Future Directions, Elsevier, Oxford. 